\title{
A Cognitive Interpretation of Negative Transfer and Forgetting of Paired Associates 1
}

\author{
JAMES G. GREENO \\ Carlton T. JAmes \\ University of Michigan, Ann Arbor, Michigan 48104 \\ Rutgers University, New Brunswick, New Jersey 08903 \\ Frank J. DaPolito \\ University of Dayton, Dayton, Ohio, 45409

\begin{abstract}
A new interpretation of negative transfer and forgetting is presented, using concepts of storage and retrieval from memory. According to the interpretation given, one process in negative transfer is interference with the storage of new items, produced by carryover of the encodings of stimuli and responses from earlier pairings. The other process in transfer, and the main process in forgetting, is interference with the retrieval process, produced mainly by the inclusion of the same stimuli in two retrieval systems. These ideas are used to interpret three empirical results. The first came from measurements of difficulty in two stages of pairedassociate memorizing that were obtained for four paradigms of negative transfer. A second finding is based on a series of different retention tests given after interpolated learning in the four paradigms. And the third result consists of evidence that recall of two responses paired with the same stimulus is independent.
\end{abstract}

The purpose of this paper is to present the outlines of a new theory of negative transfer and forgetting. The operative concepts of the theory are storage and the development of retrieval systems for items in a memorizing task.

In our perception of current theoretical analyses of negative transfer and forgetting, four general concepts seem to carry most of the explanatory burden. Three of these, response competition, associative interference, and unlearning, refer to processes that involve interactions between individual associations. Usage is not entirely consistent among theorists, but as a rough characterization we can say that response competition interferes

\footnotetext{
1 The results and opinions in this article were presented by the first author at meetings of the American Psychological Association in Washington, D.C., September, 1969. The work was supported by the U.S. Public Health Service under Grant MH12717 to Indiana University, and by the Advanced Research Projects Agency under Contract AF 49(638)-1736 with the Human Performance Centre, University of Michigan.
}

CC 1971 by Academic Press, Inc. 331 with performance of one response when both that response and another have been learned as associates of the same stimulus. Associative interference interferes with the learning of a new pairing when either the stimulus has been associated with a different response or the response has been associated with another stimulus, or both. And unlearning results in the loss of a stimulus-response association from accessible memory because its response is now paired with a different stimulus or because its stimulus is now paired with a different response. A fourth general concept is a response selector mechanism, which influences the availability in memory of sets of responses that have occurred together in lists of associations. Recent detailed discussions of these concepts, as well as thoroughly compiled bibliographies, have been given by Martin (1968) and Postman (1961).

The ideas that we will present in this paper are an extension of a theory of associative memorizing developed in earlier articles (Greeno, 1970; Humphreys \& Greeno, 1970). 
It is assumed that the process of memorizing an association involves two main subprocesses: storing a record of the stimulus-response pair in memory as a unit, and learning to retrieve the item from memory when the stimulus is presented on a test. The evidence that led to this conceptualization was obtained by measuring the difficulty of two hypothetical stages of learning, using as measures estimates of the parameters of a Markov model. (A brief description of the statistical techniques will be presented in the second section of this paper.) The gist of the results was that stimulus similarity, as well as response difficulty, had marked effects on the difficulty of accomplishing the first stage of learning, but the second stage of learning was affected only by stimulus similarity, not by response difficulty. This conclusion seems quite compatible with the idea that the first stage of learning consists of storing a relatively permanent record of the stimulus-response pair in memory, and the second stage involves learning to retrieve the stored record reliably when the stimulus is presented on a test (Humphreys \& Greeno, 1970). The result seems rather improbable on the more popular assumption that the two stages of memorizing are response learning and the learning of an associative hookup (Underwood \& Schultz, 1960).

If it is accepted that the process of memorizing an association mainly involves storing the pair in memory and learning to retrieve it, rather than the acquisition of responses and the learning of stimulus-response hookups, then some doubt is cast on the main concepts currently being used to explain negative transfer and forgetting. The concepts of response competition, associative interference, unlearning, and a response selector mechanism were developed in a framework including the idea that learning an association is mainly a process of acquiring a response pool and forming connections between stimuli and their responses. Questioning that assumption about the learning process is tantamount to questioning the relevance of concepts based on that assumption to the processes involved in negative transfer and forgetting. Therefore, one of the purposes of this paper is to present an interpretation of negative transfer and forgetting that is consistant with the hypotheses about memorizing that we have been led to in previous investigations.

On the other hand, the theory that we will present has been developed in the light of several empirical findings that we will present in this article. A number of these empirical results are difficult to explain using the currently popular assumptions about negative transfer and forgetting that we have been referring to, and we therefore believe that the data we will present add to the weight of evidence favoring the general interpretation of memorizing and forgetting based on storage and retrieval mechanisms.

\section{THEORY}

Although the theory that we will present grew directly out of the empirical findings that we will present later, we will describe our theoretical cart before presenting the empirical horses that led us to it. We hope that this order of presentation will help clarify the significance that we now see in the empirical results.

First, one process in negative transfer is interference with the storage of new items. The most likely source of this interference is the influence of past encodings of stimuli and responses carried over from earlier pairings. A second process contributing to negative transfer is interference with the process of learning to retrieve stored items from memory. This probably is produced mainly by stimulus members of pairs presented during transfer that have been used as retrieval cues for items in a preceding list, but there is some evidence suggesting that response carry-over also influences the process of learning to retrieve. The process mainly responsible for forgetting is interference with retrieval systems. This process can be thought of as the complement 
of the interference in learning to retrieve new items that is assumed to contribute to negative transfer. A retrieval system based on a set of stimuli will interfere with the development of a new retrieval system based on the same stimuli, and the development of the new system will result either in a suppression of the first system, or in some cases, a partial breakdown of the first system.

We will elaborate on these assumptions in turn. First we will consider the process of storing a record of a paired-associate and the mechanism that seems most probably to interfere with storage.

The notion of storing a pair as a unit has been proposed rather consistently by Gestalt theorists (Köhler, 1941 ; Asch, 1969). According to this idea, the process of storing a pair in memory consists of finding or generating a property of the stimulus-response pair-in other words, a relational property of the stimulus and response that makes them a mental unit. The importance of relational properties has been supported by Asch's (1969) work, showing that two properties are easier to associate when they are embedded in a single figure than when they are spatially separated, and by Bower's (1971) extensive studies of elaborative coding strategies showing that success in memorizing is greatly enhanced when $S$ s are trained to form mental images or sentences that incorporate relationships between the members of pairs. However, it should be emphasized that the concept of "relation" is very general and is not restricted to cases where $S$ uses a specific strategy for generating relational properties. If a pair is memorized by rote, without the aid of a special mnemonic link, we would still say that a relation had been stored in memory. In such a case, the relation between the stimulus and response might simply be their common membership in a pair that $S$ stored in memory as a unit. From $S$ 's point of view, such relationships probably seem more arbitrary than those that involve explicit properties, images, or other elaborative structures, and the available data suggest that they are also less memorable.

An emphasis on relational properties of associative learning is relevant to the issues of this paper because of the implication that the encodings of stimuli and responses in memory are interdependent. The issue between associationistic and Gestalt views has always been an issue between "nothing but" and "something more." In this particular case, "nothing but" means just a connection between otherwise independent mental entities. On the simpler associationistic view, there are representations of each stimulus and response in memory, and there are connections between appropriate elements. The "something more" that is included in the Gestalt view is the idea that because the basis of memory storage is relational, the representation of each stimulus in memory is influenced by the response that it is paired with, and similarly the representation of each response is influenced by its stimulus.

The theory that we propose uses a rather sharp distinction between retention of a pair, which is what is usually tested, and recognition of either the stimulus or response or both, which is usually not measured in a memorizing experiment. [A notable exception is in Martin's (1967) work.] It seems reasonable to assume that in most situations where $S$ studies a relatively short list of paired associates and then is given a second list where either the stimuli or the responses or both are carried over from the first list, there is a very high probability that $S$ recognizes the elements that are not new whether he is able to recall the response or not. Another way of saying this is that $S$ is very likely to encode the old stimulus or response in the same way that he did in studying the old item regardless of whether he retains the pair. We will refer to this assumption as the principle of persistent encoding.

In the present theory persistence in encoding is relevant to interference with memory storage because encodings of stimuli and responses are assumed to be interdependent. 
When the stimulus or response of a pair has been studied earlier as part of another pair, the encoding of the old element was established in a different context than the one that $S$ now has to deal with. Persistence in encoding a stimulus or response element in a way that becomes inappropriate can reasonably be assumed to produce added difficulty in generating a relationship between the elements of a new pair, and therefore should cause interference with the process of storing the new item.

The second main assumption of the present theory is that in a transfer list the occurrence of stimuli that also were in an earlier list causes interference with the process of learning to retrieve the transfer items from memory after they have been stored. This probably occurs mainly because of relationships that $S$ discovers or generates among the stimuli or stimulus-response pairs in the first list. That is, $S$ has to develop an organized system for retrieving items in order to master the first list. This process probably involves the stimuli more than the responses, since the stimuli are presented on tests and have to serve as retrieval cues. When those same stimuli occur in a transfer list, $S$ has to develop a new retrieval system, and he has increased difficulty in this because he has to either suppress the first system or reorganize his retrieval mechanism in order to be able to retrieve the transfer items efficiently.

While our main emphasis regarding interference with learning to retrieve involves stimuli that are carried over from an earlier list, there are reasons for expecting responses to have effects as well. Assumptions about these probable effects will be discussed in relation to the data that seem to require them.

The third main assumption of the present theory involves processes that result in forgetting of previously learned items. In the present theory, retroactive forgetting is explained by hypothetical mechanisms involving decreased retrievability of items from memory. We find no compelling reasons for assuming that stored representations of items are lost from memory. If stimuli that were used in a training list also occur in a transfer list, then mastery of the transfer list requires that $S$ develop a new retrieval system using those stimuli. And it is assumed that this new retrieval system will interfere with $S$ 's ability to use the stimuli as retrieval cues for the original items. There are two ways in which this interference apparently occurs. One type of interference seems to involve suppression of the original retrieval system, but not a substantial breakdown of the system. This apparently occurs when $S$ is able to differentiate relatively clearly between the retrieval systems developed in original and interpolated learning, as in the A-B, A-C transfer paradigm where the responses differ in the two lists and provide a basis for easy discrimination of the two retrieval systems. The other type of interference with retrieval of previously learned pairs involves disorganization or breakdown of the original retrieval system produced by the requirement of developing a new retrieval system in a situation that does not provide for clear differentiation between the "retrieval systems needed for original and interpolated learning, as in the A-B, A-Br transfer paradigm.

The preceding brief sketch will be filled out to some extent in the empirical sections that follow. We will now proceed to describe the empirical results that guided us in developing the hypotheses about negative transfer presented above.

\section{Negative Transfer}

Suppose that the process of memorizing a paired associate involves two subprocesses that occur in approximately a sequential fashion. Suppose further that the accomplishment of each stage is approximately an all-ornone process. Finally, suppose that the probability of correct response is zero on any item for which the first stage of learning has not been accomplished; when the first stage 
has been accomplished for an item, but the second stage has not yet been accomplished, the probability of a correct response is some positive probability $p$; and when the second stage has been accomplished, the probability of correct response is 1 .

The assumptions stated above are the assumptions of a two-stage Markov chain of a kind used by numerous investigators to analyze various kinds of learning processes (for example, Bower \& Theios, 1964; Kintsch \& Morris, 1965; Theios \& Brelsford, 1966; Waugh \& Smith, 1962). We have used the two-stage model to obtain measurements of difficulty in the two hypothesized stages of learning of transfer lists. These applications of the model, like those in earlier investigation (Humphreys \& Greeno, 1970), reflect our belief that the approximation provided by the model is close enough to an accurate description of the learning process that the measurements obtained with it will not be seriously misleading. In other words, we are not committed to the idea that learning is exactly a process of two all-or-none stages that occur sequentially. We routinely apply a series of tests of the model using the distributions of several empirical statistics and when these tests indicate that the model is acceptable, we assume that the parameter values that are estimated probably are related in reasonable ways to subprocesses of learning.

Figure 1 is a graphical representation of the model, showing the parameters that are involved most importantly in our measurements. Items are assumed to start in State 0. The first stage of learning is accomplished when an item leaves State 0, and this occurs

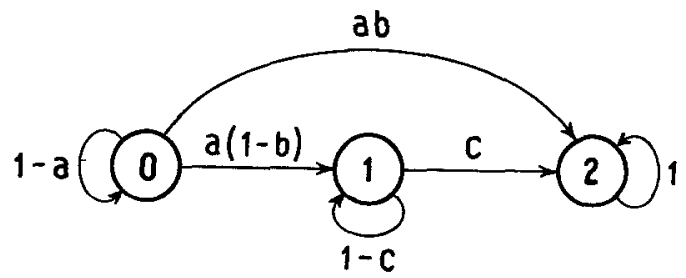

Fig. 1. Graphical representation of two-stage Markov model of memorizing individual items. with probability $a$ on each trial so the value of $a$ measures the difficulty of the first stage. The learning of an item is complete when it enters State 2. This can occur on the trial when the item leaves State 0 (the probability is $b$ ) or the item can spend some time in State 1 where the probaility of accomplishing the second stage of learning is $c$. Therefore, $b$ and $c$ measure the difficulty of the second stage. In addition to the parameters shown in Figure 1, there are other parameters that have to do with performance in the intermediate state.

The parameters of the model were estimated by the method of maximum likelihood, using the complete sequence of correct responses and errors made on each item by each $S$. We used the simplifying assumptions of equal parameter values for all $S$ s and items and independence among the items learned by a $S$. The estimation was accomplished by iterative search of the parameter space using Subroutine STEPIT (Chandler, 1965). Having obtained optimal parameter values, we checked the goodness of fit of the model to distributions of a number of statistics in the data. Data for which theoretical and empirical distributions agreed reasonably well were included in the present collection of results. A more detailed description of statistical methods is in Greeno (1968).

Rather than presenting the values of the parameters estimated for the various experimental conditions, we will present the mean number of trials taken in accomplishing each stage of learning in the various experimental conditions. These quantities are functions of the theoretical parameters and were calculated using the estimated parameter values. We choose to present the mean numbers of trials taken in accomplishing the two stages of learning, rather than the parameter values, because different simplifying assumptions about the parameters of the model were acceptable in different conditions and this makes it difficult to compare parameter values directly between different conditions. In addition, the mean number of trials taken to 
accomplish each stage seems an intuitive measure of the difficulty of each stateperhaps more so than the probability of accomplishing the stage.

The measures that we report are obtained from the estimated parameter values and bear no simple relationship to any statistics that can be easily computed from data. However, there is a strong correlation between the trials taken for the first stage and the number of errors before the first correct response on an item. And the number of trials taken for the second stage is strongly correlated with the number of trials between the first correct response and the last error on an item.

The theoretical ideas presented in the first section have some reasonably specific things to say about where we should find differences between different transfer paradigms. Interference with storage, the first stage of learning, is supposed to be due to persistence of encoding, and it is consistent with the present view to suppose that effects on storage because of stimuli should not be very different from effects on storage caused by responses. This suggests that following learning of $A-B$, the first stage of learning should be easier in a C-D list than in either an A-C or a C-B list, but that A-C and C-B should not be different from each other. The first stage of learning in A-Br might be harder than in A-C or C-B, but this expectation presupposes that stimulus and response effects on interference with storage are cumulative.

Interference in the second stage of learning is interference with learning to retrieve, and it is primarily an effect due to stimuli. Thus, the second stage of learning with a C-D or a C-B list should be easier than with an A-C or an $\mathrm{A}-\mathrm{Br}$ list. Of the two paradigms in which there is interference in the second stage, $\mathrm{A}-\mathrm{Br}$ might be harder than A-C, since the A-C condition allows the possibility of easier differentiation between the two retrieval systems that $S$ must develop for the two lists.

We have applied the two-stage Markov model to the data from several experiments involving the transfer paradigms mentioned above. One study involving all four paradigms was conducted by James (1968). Judith Goggin kindly permitted us to analyze unpublished data from a study that included all but the C-B paradigm. And several comparisons between $\mathrm{C}-\mathrm{B}$ and $\mathrm{A}-\mathrm{Br}$ were carried out by James and Greeno (1970). Materials in most of the experiments were pairs of adjectives; Goggin's study used CVCadjective pairs. List length varied from six to ten pairs, and there were other procedural variations among the experiments.

Table 1 shows the results obtained in these analyses. Most of the data available to us involve comparisons between $\mathrm{C}-\mathrm{B}$ and $\mathrm{A}-\mathrm{Br}$, and a clear and interesting result was obtained.

TABLE 1

Mean Trials Inferred for FIrst and Second Stages of Learning

\begin{tabular}{|c|c|c|c|c|c|c|c|c|}
\hline & \multicolumn{4}{|c|}{ First stage } & \multicolumn{4}{|c|}{ Second stage } \\
\hline & C-D & A-C & C-B & $\mathrm{A}-\mathrm{Br}$ & C-D & $\mathrm{A}-\mathrm{C}$ & C-B & $\mathrm{A}-\mathrm{Br}$ \\
\hline Goggin, CVC-Adj & 2.0 & 3.5 & & 3.5 & 1.6 & 2.6 & & 3.8 \\
\hline James, 4-sec, recall proc. & 1.5 & 2.0 & 1.6 & 1.8 & 1.2 & 1.4 & 0.5 & 2.0 \\
\hline $\mathrm{J} \& \mathrm{G}, 10$ items No OT & & & 3.9 & 4.5 & & & 2.8 & 4.1 \\
\hline J \& G, 10 items, OT & & & 3.5 & 6.5 & & & 3.0 & 10.1 \\
\hline J \& G, 6 items, No PT, No OT & & & 3.0 & 2.6 & & & 1.3 & 5.1 \\
\hline J \& G, 6 items, No PT, OT & & & 2.6 & 3.2 & & & 2.7 & 4.4 \\
\hline J \& G, 6 items, PT, No OT & & & 1.9 & 1.7 & & & 1.6 & 3.8 \\
\hline J \& G, 6 items, PT, OT & & & 2.5 & 2.9 & & & 1.2 & 3.6 \\
\hline
\end{tabular}


The difference in difficulty between $\mathrm{C}-\mathrm{B}$ and $\mathrm{A}-\mathrm{Br}$ is well documented in the literature, and our analyses indicate that this difference occurs almost entirely in the second stage of learning. In all of the seven comparisons, the second stage of learning in $\mathrm{A}-\mathrm{Br}$ was significantly more difficult than in C-B. However, in only one case was there a significant difference in the difficulty of the first stage.

This result is to be expected if the theory of the first section of this paper is accepted. The difference between $\mathrm{C}-\mathrm{B}$ and $\mathrm{A}-\mathrm{Br}$ is a difference in stimuli; new stimuli are presented in C-B but the first-list stimuli are present in A-Br. And the main effect of having the first-list stimuli is to produce difficulty in learning to retrieve new items. Since the present theory says that learning to retrieve is the second stage of learning, a difference in learning to retrieve should appear as a difference in the second stage of learning.

The available data are too sparse to permit firm conclusions about other comparisons, but the results that we have are consistent with the general picture implied by the present theory. Goggin's data are probably more reliable than James' for this purpose because learning took place so rapidly in all of James' conditions. Our best guess about the ordering of the four paradigms in each of the two stages is given in Eq. 1.

$$
\text { First stage: } \quad \mathrm{C}-\mathrm{D}<\mathrm{C}-\mathrm{B} \approx \mathrm{A}-\mathrm{C} \approx \mathrm{A}-\mathrm{Br}
$$

Second stage: $\mathrm{C}-\mathrm{B} \leqslant \mathrm{C}-\mathrm{D}<\mathrm{A}-\mathrm{C}<\mathrm{A}-\mathrm{Br}$ (1)

In addition to the data used in the present analyses, Eq. 1 is supported indirectly since it agrees with the overall ordering of the paradigms that is well established in the literature. When meaningful responses are used, $\mathrm{A}-\mathrm{Br}$ is the hardest transfer paradigm, and $A-C$ is somewhat easier. The $\mathrm{C}-\mathrm{D}$ and $\mathrm{C}-\mathrm{B}$ paradigms are easier than A-C, and while C-B is sometimes significantly harder than $C-D$, the difference is not obtained consistently. Further data need to be collected, particularly to see how the pattern of results changes as the meaningfulness of responses is varied. In the meantime, we believe that Eq. 1 represents a useful hypothesis about the ordering of the difficulty of learning in the four transfer paradigms. It specifies things a bit more than the previous literature, and it has at least a tentative basis in theory and data.

\section{RETROACTIVE INTERFERENCE}

In the second section we considered comparisons among the four standard transfer paradigms in the difficulty of learning the transfer lists. In this section we consider comparisons among the effects that the transfer paradigms have on the retention of first-list items. Our main findings involve a further confirmation and extension of an important discovery by Postman and Stark (1969), who found that the relative amounts of retroactive interference produced by transfer lists depend strongly on the nature of the retention test. Specifically, Postman and Stark found that with a standard test of recall, first-list items were recalled less well after interpolated learning in the A-B, A-C paradigm than after A-B, A-Br learning. However, when first-list retention was measured by a multiple-choice recognition test the ordering was reversed.

Postman and Stark's finding is consistent with the assumption about forgetting presented in the first section, where we ventured the hypothesis that in A-B, A-C learning the first-list retrieval system tends to be suppressed, but left intact, while in A-B, A-Br learning the first-list retrieval system tends to disintegrate. In addition, the present theory leads us to expect little or no forgetting following C-D or C-B interpolated learning, since the primary cause of forgetting is assumed to be interference with the first-list retrieval system, and in C-D and C-B learning there is little or no need for the new learning to interfere with the first-list retrieval system.

The data that we have to present are from James' (1968) dissertation study. James' $S$ s learned two lists, with $18 \mathrm{Ss}$ in each of four 
groups receiving A-C, C-B, A-Br, and C-D conditions. Each list consisted of 10 adjective pairs and was learned to a criterion of one perfect trial by the study-test procedure with 4-sec presentations. Four tests of retention of items from the first list were given following learning of the second list to a criterion of one perfect trial. The results are shown in Table 2.

First there was an MMFR test, where $S$ was given a sheet of paper with all the stimuli and was asked to write down both responses that had been paired with each stimulus. Recall was clearly worst after A-C transfer, and recall after $\mathrm{A}-\mathrm{Br}$ was slightly worse than

TABLE 2

Proportions of Items Remembered from First List

\begin{tabular}{lcccc}
\hline & $\begin{array}{c}\text { MMFR } \\
\text { test }\end{array}$ & $\begin{array}{c}\text { Response } \\
\text { completion }\end{array}$ & $\begin{array}{c}\text { List 1 } \\
\text { recall }\end{array}$ & $\begin{array}{c}\text { List 1 } \\
\text { matching }\end{array}$ \\
\hline A-Br & .81 & .98 & .69 & .72 \\
A-C & .57 & .72 & .70 & .94 \\
C-B & .88 & .94 & .89 & .93 \\
C-D & .88 & .89 & .93 & .97 \\
\hline
\end{tabular}

recall after C-B or C-D. Table 2 does not include performance on the second-list itemsit was nearly perfect in all conditions.

Following the MMFR test, $S$ was given a sheet of paper with the first letter and one other letter of each response and was asked to fill in the rest of the letters in each response. For example, one response was MANLY; some $S s$ had a test item MA__ and other $S$ s had a test item M__.Y. The partial cues apparently allowed $S \mathrm{~s}$ in the A-C group to remember some of the first-list responses that they had forgotten during the learning of the second list. Or, putting this in another way, some of the responses were in memory in a form that allowed their retrieval when two letters from the response were presented as a cue, but not when the stimulus was presented as a cue. Specifically, of the 77 items that were missed by A-C $S$ s on the MMFR test, 44 were given correctly on the response completion test.
In the third test $S$ was again given a sheet of paper with all the stimuli and was asked to write down only the first-list repsonses. The results are interesting in two respects. First, there was significant improvement in both the A-C and C-D groups, relative to performance on the MMFR test given immediately after second-list learning. James did not have a control for spontaneous recovery, but it still seems safe to assume that some recovery of first-list associations was brought about by the response-recall that was prompted during the response completion test. On the other hand, regaining the response on the second test did not guarantee recall on the third test. Of the 44 items whose responses were given correctly by A-C $S$ s on the response completion test after being missed on the MMFR test, 26 were missed on the third test.

The other interesting result in the third test was a significant decrease in performance by the A-Br $S$ s, relative to performance in the MMFR test. At least part of this difference was probably due to the nature of the first test, where $S \mathrm{~s}$ were asked to write down both responses to each stimulus. Many Ss wrote down the second-list responses first, thereby making it easy to avoid giving second-list responses as errors in place of first-list responses. It also seems likely that there was some loss of differentiation between the lists during performance on the response completion test.

In the final test $S \mathrm{~s}$ were shown the first-list stimuli and responses and asked to match them. Again, two results are interesting. First, A-C Ss correctly matched nearly all of the items. The improvement from the third to the fourth test was significant, even taking account of the high probability of guessing correctly on a matching test when $S$ knew several of the items. Secondly, the A-Br $S$ s failed to achieve as high a level of performance on the final matching test as they did on the initial MMFR test, which was virtually a matching test since they were able to write down nearly all the second-list responses 
correctly and the two sets of responses were the same.

It is clear from these results that the amount of forgetting produced by a particular kind of second list is not a simple quantity. As in Postman and Stark's (1969) experiment, comparisons between the amounts of forgetting produced by the different transfer lists, depend on the kind of test given. The general point is seen most clearly in comparison between $\mathrm{A}-\mathrm{Br}$ and $\mathrm{A}-\mathrm{C}$. According to the immediate MMFR test, there was considerably less forgetting in $\mathrm{A}-\mathrm{Br}$ than in A-C. However, after a procedure that permitted recovery of some first-list responses in A-C, a recall test showed virtually equal performance in the $\mathrm{A}-\mathrm{C}$ and $\mathrm{A}-\mathrm{Br}$ groups. And when all the first-list responses were shown, $S$ s in A-C performed better than $S$ s in A-Br. On the other hand, it would probably be incorrect to say that all of the improvement in the A-C group was due to an increase in availability of the first-list responses. The fact that makes an availability explanation seem implausible is the low performance on the third test on items given correctly in response completion.

The pattern of results obtained in these tests seems to fit nicely with the theory presented in the first section of this paper. The poor retention of first-list items following A-C interpolated learning with the improvement in performance after recall of some of the responses seems quite agreeable to the idea that forgetting produced by learning an A-C transfer list is largely accomplished by suppression of the first-list retrieval system. The degradation of performance in the group that had A-Br transfer suggests that these $S$ s did not have their first-list retrieval system in a form that permitted recovery of first-list retention. It is relevant to the present theory that retention following both C-D and C-B transfer was very high. This is to be expected according to the present theory, because the new stimuli permits retrieval systems for first-list items to remain relatively intact.

\section{ANALYSIS OF MMFR}

The final result that we will present was the one that led us to the hypothesis of interference with storage due to persistent encoding. The main principle needed for our empirical results is that interference produced by earlier presentations should be independent of whether $S$ can remember the item presented earlier. The principle of persistent encoding accomplishes this, since it assumes that $S$ tends to encode old stimuli or responses in new pairs the way they were encoded previously, whether or not the old pair can be retrieved from memory. Thus, if the conditions of an experiment permit $S$ to retain his encoding of a stimulus or response that is used both in training and transfer, the amount of interference produced by that overlapping element will be independent of $S$ 's recall of the first-list item.

Persistent encoding produces interference with storage. Interference with learning to retrieve and with retrieval is assumed to occur because organized systems of retrieval either are suppressed or disintegrate. On this assumption, interference with the second stage of learning and with retrieval of learned items is due to a process operating at the level of several items or the whole list, rather than at the level of individual items. Therefore, the present assumption permits the possibility that the amount of interference with retrieval will be independent of whether $S$ can recall individual items.

These hypotheses about interference constitute our tentative explanation of a finding that DaPolito (1966) obtained in his dissertation studies, and that seems to be quite ubiquitous. The finding has to do with performance on an MMFR test. Let $R_{1}$ and $R_{2}$ be the two responses paired with a given stimulus in an $\mathrm{A}-\mathrm{B}, \mathrm{A}-\mathrm{C}$ or an $\mathrm{A}-\mathrm{B}, \mathrm{A}-\mathrm{Br}$ transfer paradigm. The amounts of retroactive and proactive interference are usually measured by the marginal proportions $\mathbf{P}\left(\mathbf{R}_{1}\right)$ and $\mathbf{P}\left(\mathbf{R}_{2}\right)$, compared with appropriate control items. The 
analyses that we are concerned with here involve the additional information obtained when the joint events $\left(\mathrm{R}_{1}, \mathrm{R}_{2}\right),\left(\mathrm{R}_{1}, \mathrm{R}_{2}\right)$, $\left(\mathrm{R}_{1}, \mathrm{R}_{2}\right)$, and $\left(\mathrm{R}_{1}, \overline{\mathrm{R}}_{2}\right)$ are examined.

To illustrate the analysis as well as the finding, we will describe DaPolito's (1966) first experiment briefly. It was patterned after Estes' (1960) miniature experiments using an RTT procedure. A number of different paired-associate items were presented for $S$ to study, and then all the items were tested, and then tested again.

Some of the stimuli appeared at two different times with different responses, producing an A-B, A-C condition in the miniature experiment. Other stimuli were presented only once, and the sequence of items was arranged so that some items served as controls for the retention of A-B and others were controls for comparison with the experimental A-C items.

The stimuli were distinctive pictures of the kind used by Polson, Restle, and Polson (1965) and the responses were the numerals 1-24. Instructions at the beginning of the experiment did not include mention of the fact that some stimuli would appear with different responses; $S$ was told to try to learn as many pairs as possible and that there would be tests later to evaluate how much $S$ had learned. Items were then presented for study for $4 \mathrm{sec}$ each, with $4 \mathrm{sec}$ between presentations. All the study presentations were given without interruption before any items were tested. Then $S$ was instructed about the test trials. $E$ remarked that some stimuli had been paired with one response, and some with two. He asked $S$ to give two responses on every test trial, selecting for his second response any of the numbers 1-24 he wished to in cases where only one response had been paired with a stimulus. The purpose of having $S$ give two responses on every test was to avoid missing responses that $S$ knew but failed to give because he thought a two-response item was only a one-response item. Thirty-six college-student $S$ s were tested. The list included eight experimental A-B, A-C items and two control items for each of the experimental responses. It also included four control items that received two study presentations each, but the two-reinforcement control items did not yield results of interest.

The results showed no retroactive interference; on the first test $P\left(R_{1}\right)$ for the A-B, A-C items was .53 compared to .54 for the controls. However, there was significant negative transfer or proactive interference; $P\left(R_{2}\right)$ was .34 compared to .58 for the comparable control items. Results on the second test were consistent with the all-or-none pattern found by Estes (1960). Probabilities of correct response on Test 2 given incorrect on Test 1 were low for all items: .08 and .04 for the experimental $R_{1}$ and $R_{2}$ responses, .06 and .10 for the two sets of control items. Retention between tests was quite high for $R_{1}$ experimental items and for control items. Probabilities of correct response on Test 2 given correct on Test 1 were .96 for experimental $\mathbf{R}_{1}$ items and were .97 and .90 for the two sets of control items. On the other hand for experimental $\mathrm{R}_{2}$ items, $\mathrm{P}\left(\mathrm{C}_{2} \mid \mathrm{C}_{1}\right)$ was only .78. This suggests that the deficit in performance of these items could have been mainly an effect of proactive interference, rather than a negative transfer effect.

But the result of main interest here involves the frequencies of joint events. Table 3 has these for both tests of the experimental items, along with the expected frequencies calculated for the chi-square test of independence. The

\section{TABLE 3}

FREQUENCIES OF JOINT EVENTS IN MMFR AND EXPECTED FREQUENCIES FOR TEST OF INDEPENDENCE

\begin{tabular}{|c|c|c|c|c|c|}
\hline & \multicolumn{2}{|c|}{ First test } & & \multicolumn{2}{|c|}{ Second test } \\
\hline & $\mathbf{R}_{2}$ & $\overline{\mathbf{R}}_{\mathbf{2}}$ & & $\mathbf{R}_{2}$ & $\overline{\mathbf{R}}_{2}$ \\
\hline $\mathbf{R}_{1}$ & .20 & .33 & $\mathbf{R}_{1}$ & $\begin{array}{l}.16 \\
(.16)\end{array}$ & $\begin{array}{r}.39 \\
\quad(.39)\end{array}$ \\
\hline$\overline{\mathbf{R}}_{1}$ & .14 & $\begin{array}{l}.33 \\
(.31)\end{array}$ & $\overline{\mathbf{R}}_{1}$ & .12 & .32 \\
\hline
\end{tabular}


data are completely in accord with the hypothesis of independence: for the first test, $\chi^{2}(1)=1.69$, and for the second test, $\chi^{2}(1)=$ 0.09 .

The finding that responses are independent in MMFR was surprising and, we believe, of considerable importance for the theory of forgetting. Therefore, a stronger check on its validity was carried out. This second experiment used a sequence of study presentations containing A-B, A-C items along with controls with only one presentation as before. However, there were different numbers of A-B presentations preceding the A-C presentation. Three items received one presentation of $A-B$, three had the $A-B$ item presented twice, and three items had three presentations of $A-B$ before the A-C item was presented. All of the A-B presentations of all items were given before any of the A-C presentations, and the spacing of the A-B presentations was balanced with respect to position in the sequence of study presentations. There were three control items with only one response for each of the six A-B conditions, and six control items comparable to the A-C items.

The stimuli were three-letter English monosyllables taken from Appendix D of Underwood and Schulz (1960). The responses were the numerals $1-30$. Sixty college-student $S$ s were tested. Instructions and other procedures were identical to those used in the experiment described above.

This experiment provides a strong test of the independence of responses because with differing numbers of presentations, A-B responses had considerable variation in probability. On the first test, $P\left(R_{1}\right)$ was .49 , .73 , and .82 for items with one, two, and three A-B presentations. The corresponding values on the second test were $.46, .69$, and .83 . If $\mathrm{P}\left(\mathrm{R}_{2} \mid \mathrm{R}_{1}\right)$ and $\mathrm{P}\left(\mathrm{R}_{2} \mid \mathrm{R}_{1}\right)$ were not equal, the obtained variation in $P\left(R_{1}\right)$ should have been large enough to give a detectable effect on $\mathbf{P}\left(\mathbf{R}_{2}\right)$. However, no detectable effect appeared. The values of $\mathbf{P}\left(R_{2}\right)$ on the first test were .30, .29 , and .32 for items with one, two, and three
A-B presentations, respectively. And the corresponding values on the second test were $.30, .26$, and .33 . The independence of responses within sets that was obtained in the first experiment was also observed here; there were six sets of data to be checked for independence, and the sum of the six chisquare statistics was $\chi^{2}(6)=4.99$.

As was the case in the first experiment, there was a substantial difference in retention between the A-C responses and their controls; the proportions of control items recalled were .48 on the first test and .43 on the second test. Another result consistent with the first experiment was a lack of evidence for retroactive interference. The average difference between $P\left(R_{1}\right)$ for the A-B, A-C items and their controls was .008 . Results that were not consistent with the findings of the first experiment were obtained when dependencies between tests were examined. The first data were consistent with the idea of a sharp threshold of performance, but the data of this experiment showed substantial proportions of correct response on Test 2 given incorrect on Test $1 ; P\left(C_{2} \mid I_{1}\right)$ was as high as .208 , and the average of the values from the various conditions was .126 , nearly four times the value expected by chance guessing.

According to the present theory, there are mechanisms of negative transfer and forgetting operating both on storage and retrieval. There are two considerations that make it probable that the interference occurring in DaPolito's situation was mainly interference with storage. One reason is theoretical; as we have formulated the process of interference with retrieval, it operates at the level of sets of items. While a certain amount of organization must have occurred in DaPolito's experiment, the continuous presentation of all study trials before any of the tests probably prevented the development of well-defined retrieval systems that could interfere with each other. The second reason is partly empirical; if interference occurred between retrieval systems, we would expect some retroactive forgetting as well as 
difficulty in remembering the A-C responses. However, there was no detectable difference between retention of A-B responses and that of their control items.

The idea that interference in DaPolito's situation was primarily located in the storage phase of learning is supported further by the result of another experiment. In this study, the picture-numeral pairs used in the first experiment were used again. Six A-B, A-C items and six control items for each of the experimental responses were included in a list that was presented to 45 college-student $S$ s for study. After the study presentations, $S$ received a series of two-choice recognition tests. Each test had a stimulus picture with two numbers, one of which had been paired with the stimulus. All of the response alternatives were taken from the set of numerals used during the study presentations. This procedure makes it possible to test retention for the two responses of an A-B, A-C item on completely separate tests, and therefore it would seem to eliminate any vestiges of response competition that might operate on a standard MMFR test. In addition, since the test involves a recognition process, there should be less demand on $S$ 's ability to retrieve items and performance should be determined primarily by the success of $S$ 's efforts to store items in memory.

The results of this experiment were similar to those of DaPolito's other experiments. There was a substantial proactive effect and little or no retroactive forgetting. The proportion of correct response was .86 for both sets of control items. For the experimental items, $P\left(R_{1}\right)$ was .83 and $P\left(R_{2}\right)$ was .68. Also, the responses were independent as in the other studies, showing that the probability of recognizing A-C was not affected by whether $S$ had succeeded in storing a record of A-B.

The independence of responses could be taken as evidence for a process involving development of separate sets of responses, with a selector mechanism determining which of the response sets should be searched for the answer at any given time (Postman, 1963).
The response-set explanation seems improbable for DaPolito's data, since there was no clear temporal separation between the sets of responses as there is in an experiment where $S$ works on two clearly separated lists. But it seems impossible for experiments in which an MMFR test is given following study of lists in the A-B, A-Br paradigm. Data for this test are not plentiful, but one study by Postman (1964) did include MMFR tests following learning of three separate pairs of lists in the $\mathrm{A}-\mathrm{B}, \mathrm{A}-\mathrm{Br}$ paradigm. The results were consistent with the finding of independence in the A-B, A-C case. The sum of three separate chi-square statistics obtained in testing independence was $\chi^{2}(3)=3.03$.

In our opinion, the independence of responses in MMFR seems to be a wellestablished general phenomenon. Figure 2 shows an empirical distribution of chi-square statistics obtained by testing independence of responses in MMFR tests from data by DaPolito (1966), Koppenaal (1963), Postman (1964), and Postman and Stark (1965). There are 25 separate tests represented, including quite a variety of interference experiments from the A-C and A-Br paradigms, verbal stimuli as well as pictures, and words as well as numerals as responses. The various results also involve a wide range in the amounts of

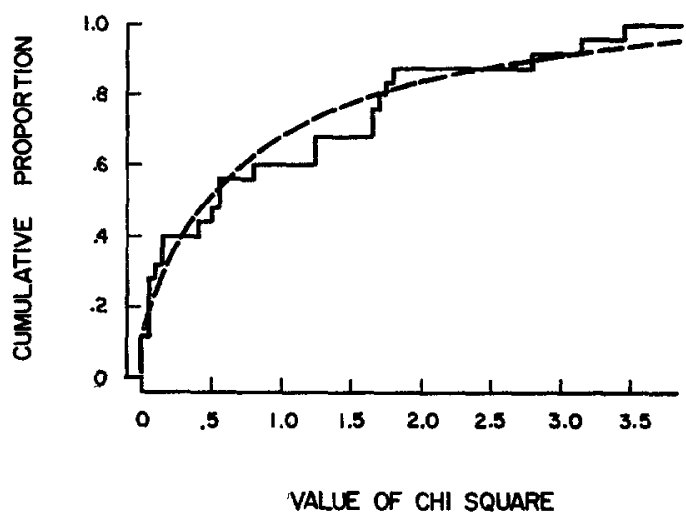

FIG. 2. Theoretical chi-square distribution and empirical distribution of chi-square statistics from 25 tests of independence of responses in MMFR. 
retroactive and proactive interference. If retention of responses in MMFR were uniformly independent, the distribution of these statistics should be chi square with one degree of freedom. This theoretical distribution is the dashed line in Figure 2, and one could hardly ask for better agreement

\section{CONCLUSION}

We have presented three empirical results. First, in analyzing the learning in different transfer paradigms, we have found evidence that in the first stage of learning, $C$-D is easier than the other paradigms, but C-B, A-C, and $\mathrm{A}-\mathrm{Br}$ are not very different from each other. In the second stage of learning, C-B and C-D are not very different, they are easier than A-C, which is easier than A-Br. Our second finding is that on an MMFR test immediately following interpolated learning, performance was considerably better if the interpolated list was $\mathrm{A}-\mathrm{Br}$ than if it was A-C, but following a response completion task recall of first-list responses was about equal in the two conditions, and finally, performance on a matching test was superior in the A-C condition. In groups receiving $\mathrm{C}-\mathrm{B}$ and $\mathrm{C}-\mathrm{D}$ interpolated lists, performance was very high on all tests. Our third finding is that in an MMFR test, performance of two responses paired with a single stimulus is independent. Apparently this is true whether the two responses occurred in separate, well-defined lists or in a series of continuous presentations, and it is true for both the A-C and the A-Br paradigms, and it is true whether tests are given by a recall or a recognition procedure.

We have presented a theory of negative transfer and forgetting that we believe is consistent with these findings. The theory considers processes of interference with storage of items and learning to retrieve items from memory and suppression and disintegration of retrieval systems, rather than processes of response competition, associative interference, unlearning, and availability of sets of responses. Neither our ideas nor the more traditional concepts are specific enough to permit definitive tests. However, we have found the results presented here hard to assimilate into the structure of concepts provided by interference theory, while concepts that seem relatively natural in the framework of storage and retrieval mechanisms make those ideas consistent with the findings. We have remarked on the features of the new theory that make it consistent with the empirical results as we went along. Perhaps a few comments now about why we find it hard to interpret the results using the more traditional concepts will provide a helpful contrast between the two ways of thinking about transfer and forgetting.

First, we find it hard to reconcile the finding of independence between responses with the notions of associative interference and unlearning. Our understanding of these ideas involves a process of replacement, with old associations making it hard to learn new ones, and the unlearning of old associations clearing the way for the learning of new pairings. To the extent that these processes are working, we would expect strong associations to cause $S$ more of a problem than weak ones, leading to a negative dependency between responses. These ideas also seem inconsistent with the pattern of difficulty found in the two stages of transfer learning. The comparison between $\mathrm{C}-\mathrm{B}$ and $\mathrm{A}-\mathrm{Br}$ is the clearest, both because it should be uncomplicated by response learning and because in our data we have the largest number of comparisons between these two conditions. If the two stages of learning were unlearning the old associations followed by replacement of them by the new pairings, we would expect the main difference between $\mathrm{C}-\mathrm{B}$ and $\mathrm{A}-\mathrm{Br}$ to be in the first stage of learning, rather than in the second, and we found the main difference to be in the second stage. Final: $y$, if the amount of forgetting of first-list associations depended only on the amount and kind of interpolated learning, then performance on first-list re- 
tention tests should show some stable ordering depending on the kind of interpolated list used, rather than the changes in ordering that have been found to depend on the kind of retention test used.

The idea of response competition is not refuted as clearly by these data. If it could be assumed that the interfering effect of a response was an increasing function of its strength, then the independence of responses and the locus of difference between $\mathrm{C}-\mathrm{B}$ and $\mathrm{A}-\mathrm{Br}$ learning would be as hard to reconcile with the idea of response competition as with the ideas of associative interference and unlearning. The usual assumption, though, is that response competition interferes with recall most when responses are equal in strength. This assumption seems to be consistent with some of the findings presented in this paper. On the other hand, if it is assumed that most of the forgetting that occurred in these studies was due to response competition, then it has to be taken as puzzling that substantial amounts of forgetting were found when retention was tested using the MMFR procedure. The MMFR test was designed to provide a situation relatively free of response competition, and the argument that response competition should not operate when $S$ is asked to give both responses still seems a valid one. And DaPolito's finding of substantial proactive interference when retention was tested by a two-choice recognition procedure makes this point even stronger.

Finally, it is hard to use the idea that forgetting occurs mainly because of interference between sets of responses to explain the forgetting that occurred in DaPolito's mixedlist experiments, and particularly in Postman's (1964) A-B, A-Br conditions, in which independence of responses was found just as it is in all other cases.

While we feel that the findings presented here are encouraging with regard to using concepts of storage and retrieval to interpret negative transfer and forgetting, we are well aware that a theoretical difference as broad as that between interference theory and a more cognitive interpretation will not be settled on the basis of a few pieces of evidence. In our judgment, choice between conceptual frameworks should probably be made by seeing which one gives a more coherent explanation of results from all kinds of memory experiment, and which one fits most easily with the best available theories of other cognitive processes such as perception and thinking. A discussion of these general issues is obviously well beyond the scope of the present article. However, we hope that we have established the feasibility of interpreting phenomena of negative transfer and forgetting of associations in a way that departs from the interpretation given by interference theory.

\section{REFERENCES}

Asch, S. E. A reformulation of the problem of associations. American Psychologist, 1969, 24, 92-102.

BOWER, G. H. Mental imagery and associative learning. In L. Gregg (Ed.), Cognition in learning and memory. New York: Wiley, 1971.

Bower, G. H., \& Theios, J. A learning model for discrete performance levels. In R. C. Atkinson (Ed.), Studies in mathematical psychology. Stanford: Stanford Univ. Press, 1964. Pp. 1-31.

ChandLer, J. P. Subroutine Stepit. Program QCPE 66. Quantum Chemistry Program Exchange, Indiana University, Bloomington, Ind., 1965.

DAPolrto, F. J. Proactive effects with independent retrieval of competing responses. Unpublished doctoral dissertation, Indiana University, 1966.

ESTES, W. K. Learning theory and the new "mental chemistry." Psychological Review, 1960, 67, 207-223.

GREENO, J. G. Identifiability and statistical properties of two-stage learning with no successes in the initial stage. Psychometrika, 1968, 33, 173-215.

GREeNO, J. G. How associations are memorized. In D. A. Norman (Ed.), Models for memory. New York: Academic Press, 1970. Pp. 257-284.

HumpHReYs, M., \& GREENo, J. G. Interpretation of the two-stage analysis of paired-associate memorizing. Journal of Mathematical Psychology, 1970, 7, 275-292.

JAMES, C. T. A consideration and rejection of the associative theory of interference and forgetting. Unpublished doctoral dissertation, Indiana University, 1968.

JAMEs, C. T. \& Greeno, J. G. Effect of A-B overtraining on A-Br. Journal of Experimental Psychology, 1970, 83, 107-111. 
KinTSCH, W., \& MORRIS, C. J. Application of a Markov model to free recall and recognition. Journal of Experimental Psychology, 1965, 69, 200-206.

KöHLER, W. On the nature of associations. Proceedings of the American Philosophical Society, 1941, 84, 489-502.

KoppenaAl, R. J. Time changes in the strengths of A-B, A-C lists: spontaneous recovery? Journal of Verbal Learning and Verbal Behavior, 1963, 2, 310-319.

Martin, E. Relation between stimulus recognition and paired-associate learning. Journal of Experimental Psychology, 1967, 74, 500-505.

Martin, E. Stimulus meaningfulness and pairedassociate transfer: an encoding variability hypothesis. Psychological Review, 1968, 75, 421441.

Polson, M. C., Restle, F., \& Polson, P. C. Association and discrimination in paired-associates learning. Journal of Experimental Psychology, $1965,69,47-55$.

Postman, L. The present status of interference theory. In C. N. Cofer (Ed.), Verbal learning and verbal behavior. New York: McGraw-Hill, 1961. Pp. 152-179.
Postman, L. Does interference theory predict too much forgetting? Journal of Verbal Learning and Verbal Behavior, 1963, 2, 40-48.

Postman, L. Studies of learning to learn II: changes in transfer as a function of practice. Journal of Verbal Learning and Verbal Behavior, 1964, 2, 437-447.

Postman, L., \& Stark, K. The role of response set in tests of unlearning. Journal of Verbal Learning and Verbal Behavior, 1965, 4, 315-322.

Postman, L., \& STARK, K. Role of response availability in transfer and interference. Journal of Experimental Psychology, 1969, 79, 168-177.

Theios, J., \& Brelsford, J. W., JR. Theoretical interpretations of a Markov model for avoidance conditioning. Journal of Mathematical Psychology, $1966,3,140-162$.

UNDERWOOD, B. J., \& SCHULZ, R. W. Meaningfulness and verbal learning. New York: Lippincott, 1960.

Waugh, N. C., \& Smith, J. E. K. A stochastic model for free recall. Psychometrika, 1962, 27, 141-154.

(Received January 14, 1971) 